\title{
The role of reactive oxygen species in cognitive impairment associated with sleep apnea
}

\author{
LINHAO XU ${ }^{1-3}$, YIBO YANG ${ }^{4}$ and JIAN CHEN ${ }^{2}$ \\ ${ }^{1}$ Department of Cardiology, Affiliated Hangzhou First People's Hospital, Zhejiang University School of Medicine, Hangzhou, \\ Zhejiang 310006; ${ }^{2}$ Department of Pathology, School of Basic Medical Sciences and Forensic Medicine, Hangzhou Medical \\ College, Hangzhou, Zhejiang 310053; ${ }^{3}$ Translational Medicine Research Center, Affiliated Hangzhou First People's Hospital, \\ Zhejiang University School of Medicine, Hangzhou, Zhejiang 310006; ${ }^{4}$ College of Chemistry and Molecular Engineering, \\ East China University of Science and Technology, Shanghai 201424, P.R. China
}

Received August 26, 2019; Accepted February 7, 2020

DOI: $10.3892 /$ etm.2020.9132

\begin{abstract}
Obstructive sleep apnea (OSA), a common breathing and sleeping disorder, is associated with a broad range of neurocognitive difficulties. Intermittent hypoxia (IH), one major characteristic of OSA, has been shown to impair learning and memory due to increased levels of reactive oxygen species (ROS). Under normal conditions, ROS are produced in low concentrations and act as signaling molecules in different processes. However, IH treatment leads to elevated ROS production via multiple pathways, including mitochondrial electron transport chain dysfunction and in particular complex I dysfunction, and induces oxidative tissue damage. Moreover, elevated ROS results in the accumulation of unfolded or misfolded proteins in the endoplasmic reticulum (ER) and increased activity of peroxisomes, such as NADPH oxidase, xanthine oxidase and phospholipase A2. Furthermore, oxidative tissue damage has
\end{abstract}

Correspondence to: Professor Jian Chen, Department of Pathology, School of Basic Medical Sciences and Forensic Medicine, Hangzhou Medical College, 481 Binwen Road, Hangzhou, Zhejiang 310053, P.R. China

E-mail: chenjian@hmc.edu.cn

Abbreviations: BACE1, $\beta$-secretase 1; BDNF, brain-derived neurotrophic factor; CPAP, continuous positive airway pressure; CREB, cyclic AMP response element-binding protein; ETC, electron transport chain; ER, endoplasmic reticulum; ERO-1, endoplasmic reticulum oxidoreductin; GSH, glutathione; GM, gray matter; GADD153, growth arrest and DNA damage-inducible gene 153; HIF- $1 \alpha$, hypoxia inducible factor- $\alpha$; $\mathrm{OH} \bullet$, hydroxyl; IH, intermittent hypoxia; Mfn2, mitochondrial fusion protein-2; NO, nitric oxide; NOS, nitric oxide synthase; OSA, obstructive sleep apnea; $\mathrm{O}_{2}{ }^{-}$, oxygen ions; PLA2s, phospholipases A2; PDI, protein disulfide isomerase; ROS, reactive oxygen species; $\mathrm{O}_{2}{ }^{--}$, superoxide radicals; $\mathrm{UPR}$, unfolded protein response; $\mathrm{XO}$, xanthine oxidase

Key words: obstructive sleep apnea, intermittent hypoxia, reactive oxygen species, endoplasmic reticulum, unfolded protein response, superoxide dismutase been found in regions of the brains of patients with OSA, including the cortex and hippocampus, which are associated with memory and executive function. Furthermore, increased ROS levels in these regions of the brain induce damage via inflammation, apoptosis, ER stress and neuronal activity disturbance. The present review focuses on the mechanism of excessive ROS production in an OSA model and the relationship between ROS and cognitive impairment.

\section{Contents}

1. Introduction

2. ROS formation

3. ROS-induced consequences lead to cognitive impairment in sleep apnea

4. Conclusion

\section{Introduction}

Obstructive sleep apnea (OSA) is a common breathing and sleeping disorder, the primary cause of which is aspiratory collapse of the pharyngeal airway, and leads to intermittent hypoxia (IH) (1). Previous studies have shown that OSA is associated with behavioral and neuropsychological deficits, including impaired spatial learning memory and cognition $(2,3)$. However, the specific mechanisms underlying the chain of events from the development of IH to cognitive impairment remain elusive. It has been suggested that reactive oxygen species (ROS), which are produced in excess in cases of IH, are strongly associated with the presence of IH-induced cognitive impairment $(4,5)$.

ROS are essential for many biological processes and these molecules are constantly formed in cells and removed by antioxidant defenses (6). Moreover, previous studies have demonstrated that increased oxidative stress is present in OSA (7-10). It has been shown that there is increased ROS production in stimulated neutrophils from patients with OSA, while the levels of ROS are attenuated after continuous positive airway pressure (CPAP) treatment $(7,8)$. Furthermore, lipid peroxidation and protein carbonylation, both of which 
are markers of oxidative stress, are observed in patients with OSA $(9,10)$. Moreover, these processes damage biomolecules such as lipids, proteins and DNA (6).

The aims of the present review were to examine the production of ROS and its role in the pathobiology of cognitive impairment in an OSA model and to investigate the underlying mechanism of ROS-induced cognitive deficiencies.

\section{ROS formation}

The ROS generated in cells include hydrogen peroxide $\left(\mathrm{H}_{2} \mathrm{O}_{2}\right)$, oxygen ions $\left(\mathrm{O}_{2}^{-}\right)$, and hydroxyl and superoxide radicals $\left(\mathrm{OH}^{\bullet}\right.$ and $\left.\mathrm{O}_{2}{ }^{\circ}\right)(11)$. Although mitochondria are described as the major source of endogenous ROS generation, other subcellular compartments, including the cellular membrane, peroxisomes and the endoplasmic reticulum (ER), are involved in ROS generation and scavenging (12-15). This section of the review will focus on the ROS generation process in different subcellular compartments and its responses under IH conditions.

Mitochondria and ROS generation. As described above, the majority of ROS are generated from mitochondria during ATP synthesis, which supplies energy for physiological functions (Fig. 1A) (16). During this process, electrons flow along the mitochondrial electron transport chain (ETC) and protons are translocated from the mitochondrial matrix into the intermembrane space, thus creating a proton gradient (4). During normal aerobic respiration, approximately $2 \%$ of electrons 'leak' from the flow, resulting in the formation of $\mathrm{O}_{2}{ }^{-}(17) . \mathrm{O}_{2}{ }^{-}$is easily detoxified by superoxide dismutase, which leads to the formation of $\mathrm{H}_{2} \mathrm{O}_{2}$ (18). While $\mathrm{H}_{2} \mathrm{O}_{2}$ is further reduced to $\mathrm{H}_{2} \mathrm{O}$ by glutathione peroxidase. $\mathrm{H}_{2} \mathrm{O}_{2}$ can also form highly reactive $\mathrm{OH} \bullet$ by reacting with metal ions, which may be responsible for oxidative stress-induced cellular damage (19).

During mitochondrial respiration, some oxidoreductases affect ROS production, including complex I via complex IV (4). Under physiological conditions, a low concentration of ROS is maintained by the action of these oxidoreductases. However, increased ROS production can be caused by the inhibition of complex I activity in the mitochondrial ETC, under IH conditions (20). Furthermore, complex I, the first oxidoreductase in the ETC, is dependent on NADH-producing substrates to produce $\mathrm{O}_{2}{ }^{-}(21)$. A previous study demonstrated that $\mathrm{IH}$ induced complex I inhibition via the upregulation of NADPH oxidase (NOX) in PC12 cell cultures (22). Moreover, when complex I activity is decreased, ROS levels are increased in mitochondria due to the inability of electrons to be transported, which results in the formation of superoxide via a one-electron reduction of oxygen (23). However, complex III, another major producer of superoxide and ROS within the mitochondrial ETC, is unaffected (24). These results suggest that complex I, and not complex III, may be involved in increasing ROS levels (Fig. 1B).

ER and ROS generation. The ER is a large organelle that participates in the correct assembly and folding of nascent proteins and is also the site of post-translational protein modifications in ATP-dependent chaperone-mediated processes (25). Furthermore, approximately $25 \%$ of ROS are derived from the ER and are primarily required for oxidative protein folding (26). Correct protein folding involves the formation of disulfide bonds in a process driven by protein disulfide isomerase (PDI) and ER oxidoreductin (ERO-1) (27). In this process, electrons are transferred from PDI to $\mathrm{O}_{2}$, resulting in $\mathrm{H}_{2} \mathrm{O}_{2}$ formation (Fig. 1A) (27).

To perform these functions, the ER lumen possesses a unique environment composed of molecular chaperones, folding enzymes and high concentrations of ATP and calcium (28). In addition, the oxidative environment favors protein folding and, in particular, the formation of intra- and intermolecular disulfide bonds (29). However, increased ROS production may lead to a loss of ER homeostasis and the subsequent accumulation of misfolded proteins, a process known as ER stress (30). Under conditions of ER stress, additional misfolded or unfolded proteins are synthesized, leading to the depletion of glutathione (GSH) (31). After GSH is utilized, the oxidizing environment facilitates the reoxidation of protein thiols through interaction with ERO-1/PDI (27). These steps produce repetitive cycles of disulfide bond breakage and formation, with each cycle generating additional ROS as a byproduct (25). Furthermore, the accumulation of unfolded proteins in the ER elicits $\mathrm{Ca}^{2+}$ leakage into the cytosol, thus causing increased ROS production in the mitochondria (Fig. 1B) (29).

NOX. NOX is a multisubunit enzymatic complex that is localized to both cellular and subcellular membranes (32). Furthermore, NOX catalyzes $\mathrm{O}_{2}{ }^{-}$-production via the one-electron reduction of $\mathrm{O}_{2}$ using NADPH (33). There have been $\geq 7$ NOX isoforms, including NOX1-5 and DUOX1-2, identified in this enzymatic system (34). Moreover, NOX can regulate electron flow and transfer to the outer heme, where the electron accepts $\mathrm{O}_{2}$ to form $\mathrm{O}_{2}{ }^{-}$(35).

As a complex of major ROS-generating enzymes, NOX plays an important role in the OSA model (36). NOX2 expression and ROS production have been shown to be simultaneously increased in patients with OSA (37). Furthermore, pharmacological inhibition of NOX by apocynin and NOX2 deficiency can attenuate arterial hypertension in an OSA model by suppressing ROS production (38). In addition to cardiovascular risk, the cognitive deficits induced by oxidative stress are partially mediated by excessive NOX activity during IH (39). Furthermore, mice lacking NOX activity present with a learning ability comparable to that of IH-exposed wild-type littermates, which exhibit spatial learning deficits in a water maze test (40). Collectively, these studies indicated that NOX may be involved in ROS production in a model of OSA. However, the specific mechanistic involvement of NOX in IH injury requires further investigation.

Peroxisomes and ROS generation. In addition to mitochondria and the ER, superoxide and other ROS are generated in subcellular organelles peroxisomes (Fig. 1). The primary function of these particles is the oxidative degradation of long-chain fatty acids (41). Peroxisomes are spherical or oval shaped particles with a diameter of $0.2-1 \mathrm{~mm}$ that are surrounded by a single membrane (42). Moreover, peroxisomes in mammals harbor $>100$ enzymes and other proteins, some of which are associated with OSA or IH (43).

Xanthine oxidase $(\mathrm{XO})$. $\mathrm{XO}$ is localized to the outer surface of the cellular membrane, as well as in the cytosol and 
A

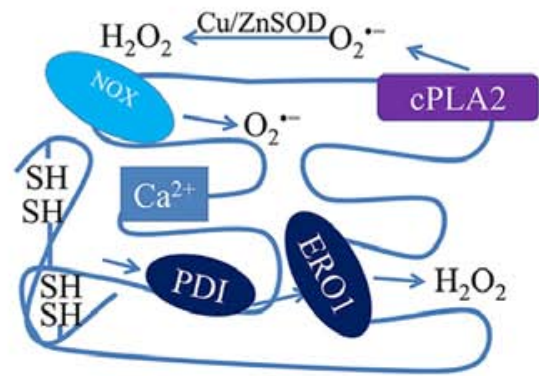

Endoplasmic reticulum

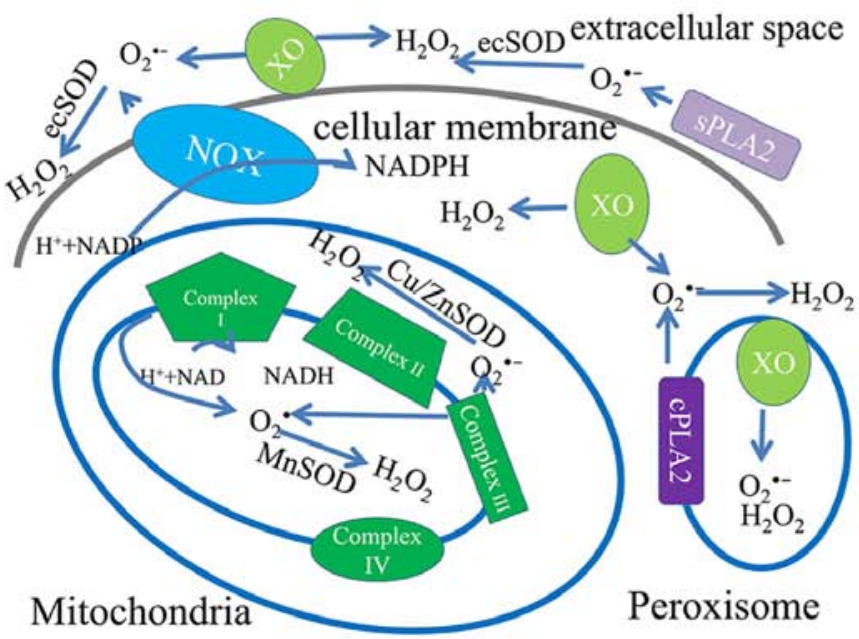

Peroxisome

B

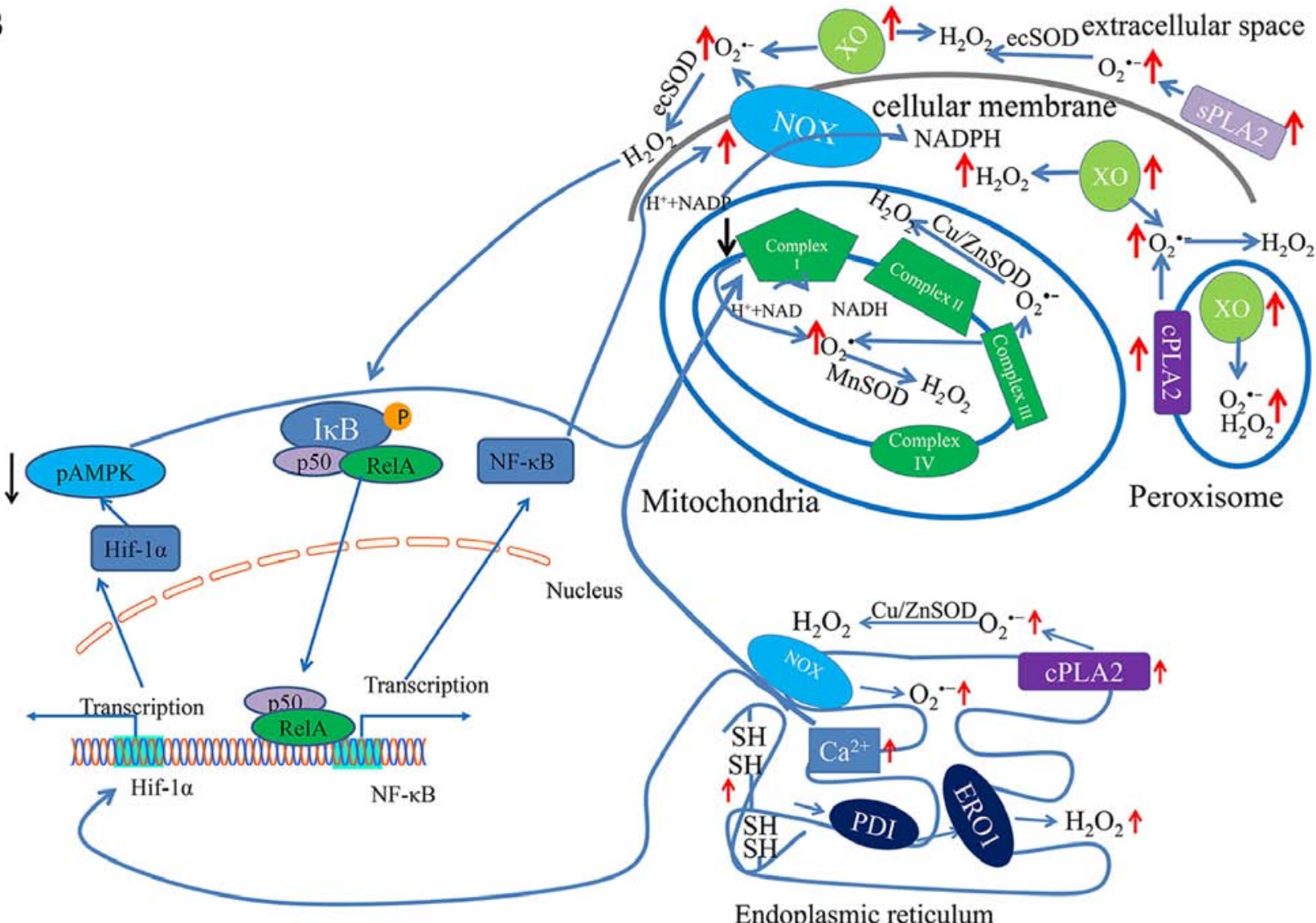

Figure 1. Mitochondria, ER and peroxisomes in the cytosol are major sites of ROS production. (A) Under physiological conditions, the electron transport chain in the inner mitochondrial membrane releases superoxide to both the matrix and the intermembrane space. NOX are localized in the cellular and ER membranes, and release superoxide towards the luminal side of the membranes. XO is localized on the outer surface of the cellular membrane, in the cytosol and in peroxisomes. XO produces both superoxide and $\mathrm{H}_{2} \mathrm{O}_{2}$. Cytosolic PLA2 is associated with the lipid layer of the cellular membrane and releases superoxide into the cytosol. Secretory PLA2 is localized in the extracellular space where it produces superoxide. (B) Under intermittent hypoxia, the activity of complex I is reduced and electrons cannot be transported, thus resulting in the formation of superoxide via the one-electron reduction of oxygen. ROS are generated in the ER as a part of an oxidative folding process during electron transfer between protein disulfide isomerase and ER oxidoreductin- 1 . Ca ${ }^{2+}$ ions released from the ER augment the production of mitochondrial ROS. The activity of certain peroxisomes, such as NOX, XO and PLA2, are upregulated to produce additional ROS. Moreover, ROS can induce the expression of hypoxia-inducible factor- $1 \alpha$ and NF- $\kappa \mathrm{B}$, which contribute to aggravated oxidative stress. Red arrows indicate increased activity or production. Black arrows indicate decreased activity. ER, endoplasmic reticulum; ERO-1, ER oxidoreductin 1; HIF-1 $\alpha$, hypoxia inducible factor; ROS, reactive oxygen species; NOX, NADPH oxidases; cPLA2, cytosolic phospholipases A2; sPLA2, secreted phospholipases A2; SOD, superoxide dismutase; $\mathrm{XO}$, xanthine oxidase.

peroxisomes (44). XO catalyzes the oxidation of xanthine to uric acid at the site of flavin adenine dinucleotide, together with the reduction of $\mathrm{NAD}^{+}$and $\mathrm{O}_{2}(45)$. In this process, the affinity for $\mathrm{O}_{2}$ (44) is significantly enhanced, resulting in univalent and divalent electron transfer to $\mathrm{O}_{2}$ to generate $\mathrm{O}_{2}{ }^{\text {- }}$ and $\mathrm{H}_{2} \mathrm{O}_{2}$, respectively (46). 
$\mathrm{XO}$ is a critical source of ROS in ischemia/reperfusion injury, which is similar to chronic cycles of hypoxia and reoxygenation (47). Moreover, IH processes are associated with elevated $\mathrm{XO}$ activity and promotion of ROS formation by increasing the proteolytic conversion of xanthine dehydrogenase to XO (48). XO has been identified to be involved in ROS production in OSA, as lipid peroxidation, a marker of oxidative stress, is reduced after the application of the XO inhibitor allopurinol (49). Furthermore, allopurinol reduces the level of ROS by decreasing free radical generation via inhibition of the $\mathrm{XO}$ system (50). These findings suggest that XO plays a key role in increased ROS production under IH conditions.

Nitric oxide (NO) synthase. NO is known to play a critical role in the regulation of cellular processes that include catalyzation by nitric oxide synthase (NOS) (51). Although excess NO can induce apoptosis via the aggravation of oxidative stress (52), the specific mechanism is not fully understood.

Moreover, two NOS enzymes, the constitutive calcium/calmodulin-dependent neuronal and endothelial isoforms (53), show sustained expression in the central nervous system. However, under certain pathological conditions, including ischemia, hypoxia and other pathological stimuli, another NOS, inducible calcium-independent isoform (iNOS), is activated (54). Furthermore, IH has been shown to augment NO generation by activating iNOS (55), which induces apoptosis via the aggravation of oxidative stress. In addition, overexpression of $\mathrm{O}_{2}^{-}$in the endothelial tissue of patients with OSA is reduced by the NOS inhibitor 1-nitroarginine methyl-ester (56). NOS has also been revealed to play a critical role in enhancing oxidative stress in OSA (56).

Phospholipases A2 (PLA2s). PLA2s belong to a family of enzymes that hydrolyze the acyl bond at the sn-2 position of phospholipids to generate free fatty acids and lysophospholipids (57). PLA2s can be classified into three families based on their calcium requirement for catalytic activity, including calcium-dependent cytosolic PLA2 (cPLA2), calcium-independent PLA2 and secretory PLA2 (sPLA2) (58). It has been shown that all these PLA2sare involved in ROS production via arachidonic acid and lipoxygenase, which produce $\mathrm{O}_{2}{ }^{--}(59)$.

While there is no direct evidence to establish a connection between PLA2 expression and IH treatment, PLA2 activity is significantly increased after ischemia/reperfusion, which is dependent on $\mathrm{Ca}^{2+}$ concentration (60). Previous studies have revealed that cPLA2 immunoreactivity is selectively higher in the hippocampal CA1 region compared with other regions in a hypoxia-ischemia rat model (61). Furthermore, inhibition of cPLA2 attenuates oxygen-glucose deprivation-induced neuronal death in the hippocampus, which suggests that cPLA2 is involved in ischemic injury (62). In addition, a biphasic increase in them RNA expression of SPLA 2 in the cortex of a rat brain after $20 \mathrm{~min}$ of transient forebrain occlusion has been shown (63). These results suggest that enhanced PLA2 activity results in the increased production of ROS in ischemia/reperfusion. Thus, it is hypothesized that the excessive ROS levels under IH conditions may also be due to increased PLA2 activity.

Misregulation of ROS-sensitive transcription factors and their downstream genes, such as hypoxia-inducible factor (HIF)-1 $\alpha$ and NF- $\mathrm{BB}(10)$, also aggravates oxidative stress (Figs. 1B and 2).
Although many organelles and peroxisomes are involved in ROS production during IH treatment, there is no direct evidence that demonstrates the underlying mechanism responsible for ROS production (64). Therefore, on the basis of previous studies, it is hypothesized that mitochondrial dysfunction may be the main source of excessive ROS production in OSA $(20,22,65)$. Thus, attenuation of mitochondrial dysfunction may be an effective strategy for future clinical therapeutics.

\section{ROS-induced consequences lead to cognitive impairment in sleep apnea}

OSA is commonly associated with cognitive impairment, particularly memory, verbal fluency, attention and perception impairments $(66,67)$, but the cause of this cognitive dysfunction is not fully understood.

Various different mechanisms that link OSA to cognitive dysfunction have been suggested (4,5,68-70). A major proposed mechanism is $\mathrm{IH}$, which induces a reduction in memory performance in patients with OSA via oxidative stress injury (71). Moreover, increased levels of ROS have been confirmed to contribute to learning and memory impairments (72). In addition, increased ROS production induced by IH can result in deficits in spatial learning and memory in rodent models, which are largely dependent on the hippocampus. Neuroimaging studies have been performed to investigate the effects of OSA (73-75). Regional gray matter (GM), which is closely associated with memory formation, is observed to be lost in multiple brain regions of patients with OSA via MRI of the cortex (75). In another study, voxel-based morphometry was performed to measure cortical GM volume and indicated that, compared with healthy control individuals, patients with OSA exhibited significant cognitive impairment and had a smaller right hippocampus (76). Therefore, the cortex and hippocampus are the two major regions associated with cognitive impairment in OSA models. Although the underlying mechanism is not fully understood, it is hypothesized that the density of capillaries and large-diameter vessels is abundant in the two regions and that these are sensitive to oxidative stress (77).

Many pathways, including mitochondrial dysfunction, inflammation, apoptosis, ER stress and neuronal activity disturbance, are proposed to be associated with cognitive impairment induced by oxidative stress (Fig. 2).

Mitochondrial dysfunction. Mitochondrial dysfunction is a common feature of patients with OSA, and the function of oxidoreductases in the mitochondrial ETC is disturbed under IH conditions (4). Furthermore, mitochondrial fusion and fission, which play important roles in mitochondrial function, have been observed in an IH model (78). Under IH conditions, mitochondrial fission occurs more frequently than fusion and leads to apoptosis through regulation of the expression of mitochondrial fusion protein-2 (Mfn2) (76). Thus, ROS may control the expression of Mfn2. While the full mechanism has not been elucidated, it is hypothesized that the mechanism could be associated with the overexpression of heme oxygenase-1, a gene that responds to oxidative stress (79). In addition, a significant correlation is found between OSA 


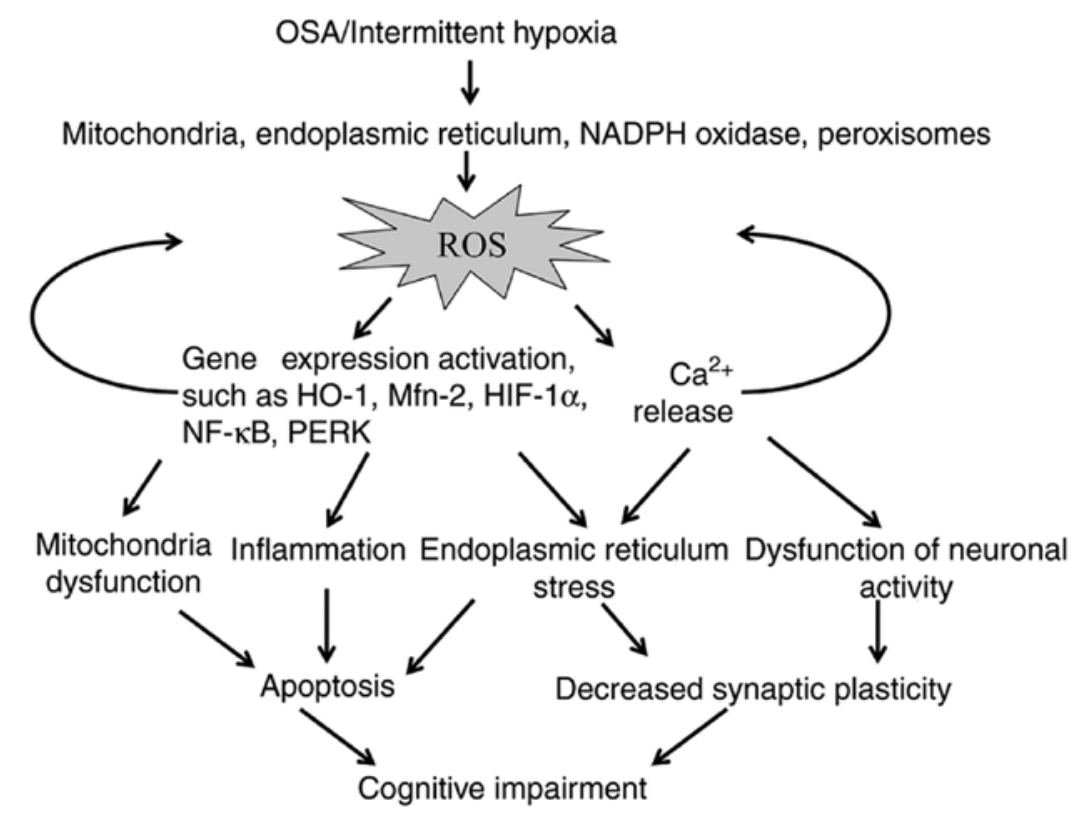

Figure 2. Proposed interactions between cognitive impairment and other pathological processes induced by increased ROS levels. Chronic intermittent hypoxia leads to increased ROS levels. Then, mitochondrial dysfunction, inflammation, ER stress and neuronal activity dysfunction are initiated by activating gene expression and $\mathrm{Ca}^{2+}$ release. These factors act together in a synergistic manner to increase apoptosis and impair synaptic plasticity, which underlie memory dysfunction. Therefore, increased ROS generation plays a pivotal role in cognitive impairment in the OSA model. ROS, reactive oxygen species; HO-1, heme oxygenase-1; Mfn2, Mitochondrial fusion protein-2; HIF-1 $\alpha$, hypoxia-inducible factor-1 $\alpha$; PERK, protein kinase RNA-like endoplasmic reticulum kinase; OSA, obstructive sleep apnea.

severity and decreased mitochondrial DNA (mtDNA) copy number, suggesting that patients with a high apnea-hypopnea index are exposed to greater systemic blood oxidative stress (65). Despite the lack of direct evidence to show that mitochondrial dysfunction is a cause of cognitive impairment in OSA, clinical research has identified that the number of copies of mtDNA are biomarkers for assessing cognitive status in neurodegenerative diseases, such as Alzheimer's and Huntington's disease $(80,81)$. The main mechanism of this effect is that mtDNA depletion induced by persistent oxidative stress leads to cognitive decline via cell apoptosis (82).

Inflammation. OSA appears to have an inflammatory component (83), but the exact mechanisms linking OSA to the inflammatory cascade are unknown. Clinically, the inflammatory component of OSA manifests as neurocognitive and behavioral deficits, with oxidative stress and inflammatory impairment, which are mediated by microglia in a model of OSA (84). Previous studies have shown that inflammation is initiated due to increased ROS production under IH cycles, as ROS induces inflammatory pathways to activate multiple proinflammatory cytokines (85). It has also been found that IH selectively activates the proinflammatory transcription factor NF- $\mathrm{\kappa B}$, which is involved in the transcription of multiple genes in the inflammation pathway (86). According to a previous study, the transcriptional activity of NF- $\mathrm{KB}$ is induced by ROS in part via the alternative phosphorylation of $\mathrm{I} \kappa \mathrm{B} \alpha$ (87). Moreover, the phosphorylation of I $\mathrm{K} \mathrm{B} \alpha$ on Tyr 42 by $\mathrm{H}_{2} \mathrm{O}_{2}$ releases a dimer that contains p50 and RelA (88). These proteins then bind to the DNA-binding domains of NF- $\mathrm{KB}$ to activate NF- $\mathrm{KB}$ transcription (89). However, the phosphorylation of RelA, which is influenced by ROS-dependent processes, leads to increased NF-KB activation (Fig. 1B) (90).
Furthermore, patients with OSA have been reported to have increased levels of NF- $\mathrm{kB}$ in neutrophils and monocytes (91). These activated cells can further contribute to oxidative stress and injury via the increased release of ROS via activation of NOX2 expression (92). Therefore, these results suggested that the underlying mechanism may include a feedback cycle involving ROS and NF- $\mathrm{\kappa B}$.

When NF- $\kappa B$ is activated, spatial learning and memory are impaired, which may be associated with declining hippocampal long-term potentiation (LTP) and dendritic branching (93). On the other hand, inflammation decreases the efficiency of the capillary system and oxygen supply to the brain, thus reducing metabolic function and oxygen intake in neurons (94). Consequently,individuals with neuroinflammation and OSA may present with cognitive deterioration.

Apoptosis. Our previous study demonstrated that rats exposed to IH show impaired spatial learning, as well as increased apoptosis in the cortex and CA1 region of the hippocampus (69), which is associated with ER stress-induced apoptosis. While the molecular mechanisms of ROS-induced apoptosis have not been fully determined, some factors have been identified. The activity of cyclic AMP response element-binding protein (CREB), which plays a critical role in neuronal survival, has been shown to decline in the hippocampal CA1 region after exposure to $\mathrm{IH}$, and is accompanied by an increase in the number of cleaved caspase-3-positive cells (95). Moreover, increased ROS levels have been reported to reduce CREB phosphorylation and decrease the expression of brain-derived neurotrophic factor (BDNF) (96). In our previous study using a chronic IH mouse model, it was found that the expression of BDNF is significantly reduced after chronic IH treatment (70). Furthermore, BDNF plays an important role in 
neuronal cell apoptosis, and increased expression of BDNF ameliorates IH-induced cognitive dysfunction by decreasing neuronal apoptosis (97).

A parallel increase in the expression of HIF-1 $\alpha$, a key regulator of cell adaptation to hypoxia, is often observed alongside the formation of ROS (98). Under IH, the $\mathrm{Ca}^{2+}$-dependent activation of calcium-calmodulin protein kinase stimulates HIF-1 $\alpha$ transcriptional activity and protein expression (99). Then, in turn, the stabilization of HIF-1 $\alpha$ promotes the synthesis of ROS in mitochondria to induce cell death via the suppression of AMP-activated protein kinase $(100,101)$. HIF-1 $\alpha$ stabilization, along with a decline in Bcl-2 and substantial caspase- 3 expression, has been observed after IH exposure (102). Furthermore, a large number of apoptotic events have been identified in rat myocardium exposed to IH (103), and the inhibition of HIF-1 $\alpha$ expression has been shown to decrease neuronal apoptosis (104). Additionally, $>50$ oxygen sensitive genes have been identified as direct targets of HIF- $1 \alpha$-mediated transactivation, such as the enzyme $\beta$-secretase 1 (BACE1) (105). It was reported that 3 days of IH treatment upregulated BACE and generated amyloid $\beta(\mathrm{A} \beta)$ via HIF-1 $\alpha$, thus compared with age-matched control individuals, patients with Alzheimer's disease have a 5-fold increased risk of presenting with OSA (106). These data suggest that inhibition of HIF-1 $\alpha$ not only suppresses apoptosis, but also reduces $\mathrm{A} \beta$ generation. Thus, HIF- $1 \alpha$ may be a potential drug target for future OSA therapy.

ER stress. ER stress induced by increased ROS expression is initiated when unfolded or misfolded proteins accumulate in the ER (30). Furthermore, ER stress induces the coordinated adaptive program known as the 'unfolded protein response' (UPR), which involves the degradation of unfolded proteins (107). The UPR consists of three independent signaling pathways, pancreatic ER kinase signaling, activating transcription factor 6 signaling and inositol-requiring enzyme 1 signaling (108). If the accumulation of toxic unfolded and misfolded proteins results in prolonged ER stress, the perturbed and overloaded ER-folding environment persists and is associated with increased cell death (109). Previous studies have demonstrated that ER stress is present in the brain in a mice model of OSA, accompanied by an increase in the expression of cleaved caspase-3, which is a protein marker of apoptosis (69). In addition, the expression of growth arrest and DNA damage-inducible gene 153 (GADD153), a proapoptotic protein activated by ER stress, is increased in the cortex and hippocampus of the OSA model, which is accompanied by cognitive impairment (69). Moreover, $\mathrm{GADD} 53^{-/-}$mice exhibit resistance to oxidative stress (110). In contrast, synaptic plasticity impairment is prevented by incubation with phenylbutyric acid (PBA), an inhibitor of ER stress (111). Furthermore, synapse degeneration is associated with elevatedGADD153 expression (112). Therefore, these studies suggest that ER stress also affects synaptic formation. Our previous study using a OSA model found that ER stress is involved in cognitive deficits due to LTP impairment in the hippocampus, as application of tauroursodeoxycholic acid, an inhibitor of ER stress, rescued LTP impairment by decreasing the number of apoptotic neurons and promoting the formation of synapses (69).
Disturbance of neuronal activity. Neuronal firing, especially robust persistent activity of neurons in the cortex and hippocampus, is critical in memory formation $(113,114)$. In a previous study, it was found that hypoxia could affect membrane excitability and may involve acute modulation of ion channels, including $\mathrm{K}^{+}$-channels, $\mathrm{Na}^{+}$-channels and $\mathrm{Ca}^{2+}$-channels, which results in the depolarization or hyperpolarization of neurons (115). Moreover, hypoxia/hypoxemia underlies several pathological processes, including neuronal activity, in these regions (116).

ROS have been implicated in LTP of neural activity as they are associated with a number of $\mathrm{K}^{+}$channels and lead to the $\mathrm{Ca}^{2+}$-dependent release of the neurotransmitter glutamate and excitotoxicity (117). ROS promote the activation of inositol 1,4,5-trisphosphate receptors in the ER, leading to $\mathrm{Ca}^{2+}$ mobilization into the cytosol, thus enhancing membrane permeability and promoting the release of glutamate (118). Furthermore, it was observed that a higher concentration of glutamate is found in the cortex of patients with OSA (119). Thus, it is hypothesized that the release of glutamate could increase neuronal excitability, and result in neuron dysfunction and apoptosis due to excitotoxicity. In addition, motor-evoked potentials, which are indicative of neuronal excitability, are higher in patients with OSA compared with healthy controls (120). Moreover, increased expression of c-Fos is observed in the cortex after IH treatment and is accompanied by increased apoptosis (102). Collectively, these previous studies have suggested that these neurons are under excitotoxic conditions, which leads to cognitive dysfunction, as measured by the Morris water maze (121). Furthermore, our previous study implanted multiple electrodes into the CA1 region of the hippocampus to monitor spontaneous discharges after chronic IH treatment. It was found that the frequency of pyramidal neuron firing in the CA1 region increased after 1-2 days of IH exposure. However, this firing decreased after 14 days of IH treatment (unpublished data). These results indicated that cognitive malfunction in the OSA model may be associated with marked cellular changes over time.

\section{Conclusion}

This review has focused on the mechanism of ROS overexpression and cognitive impairment induced by oxidative stress in IH or OSA models. While the role of ROS in cognitive impairment in OSA models is not fully understood, ROS are known to activate multiple pathways to induce neuronal dysfunction, mitochondrial dysfunction, inflammation, apoptosis, ER stress and neuronal activity disturbance $(69,80,82,117)$. These factors ultimately lead to cognitive dysfunction in the OSA model. Currently, the most commonly used methods in the clinical treatment of OSA are surgery and CPAP $(122,123)$. However, each of these methods has limitations and neither fully reverses the cognitive impairment seen in patients with OSA $(124,125)$. It is therefore important to investigate the molecular mechanisms underlying neurological impairments in patients with OSA. Moreover, identification of these molecular mechanisms will facilitate the development of targeted drug-based therapy to rescue cognitive impairment in OSA. 


\section{Acknowledgements}

Not applicable.

\section{Funding}

The present study was supported by the National Natural Science Foundation of China (grant no. 81601972) and the Natural Science Foundation of Zhejiang Province (grant no. LY19H010002).

\section{Availability of data and materials}

Not applicable.

\section{Authors' contributions}

LX conceived the idea for this review article and wrote the manuscript. YY was responsible for the manuscript revision. The manuscript was critically revised for important intellectual content by JC. All authors were involved in the writing of the manuscript. All authors have read and approved the final manuscript.

\section{Ethics approval and consent to participate}

Not applicable.

\section{Patient consent for publication}

Not applicable.

\section{Competing interests}

The authors declare that they have no competing interests.

\section{References}

1. Dewan NA, Nieto FJ and Somers VK: Intermittent hypoxemia and OSA: Implications for comorbidities. Chest 147: 266-274, 2015.

2. Vaessen TJ, Overeem S and Sitskoorn MM: Cognitive complaints in obstructive sleep apnea. Sleep Med Rev 19: 51-58, 2015.

3. Lal C, Strange C and Bachman D: Neurocognitive impairment in obstructive sleep apnea. Chest 141: 1601-1610, 2012.

4. Wang Y, Zhang SX and Gozal D: Reactive oxygen species and the brain in sleep apnea. Respir Physiol Neurobiol 174: 307-316, 2010.

5. Dayyat EA, Zhang SX, Wang Y, Cheng ZJ and Gozal D: Exogenous erythropoietin administration attenuates intermittent hypoxia-induced cognitive deficits in a murine model of sleep apnea. BMC Neurosci 13: 77, 2012.

6. Roy J, Galano JM, Durand T, Le Guennec JY and Lee JC: Physiological role of reactive oxygen species as promoters of natural defenses. FASEB J 31: 3729-3745, 2017.

7. Pilkauskaite G, Miliauskas S and Sakalauskas R: Reactive oxygen species production in peripheral blood neutrophils of obstructive sleep apnea patients. ScientificWorldJournal 2013: 421763, 2013.

8. Schulz R, Mahmoudi S, Hattar K, Sibelius U, Olschewski H, Mayer K, Seeger W and Grimminger F: Enhanced release of superoxide from polymorphonuclear neutrophils in obstructive sleep apnea. Impact of continuous positive airway pressure therapy. Am J Respir Crit Care Med 162: 566-570, 2000.

9. Vatansever E, Surmen-Gur E, Ursavas A and Karadag M: Obstructive sleep apnea causes oxidative damage to plasma lipids and proteins and decreases adiponectin levels. Sleep Breath 15: 275-282, 2011.
10. Lavie L: Oxidative stress inflammation and endothelial dysfunction in obstructive sleep apnea. Front Biosci (Elite Ed) 4: 1391-1403, 2012.

11. Zou Z, Chang H, Li H and Wang S: Induction of reactive oxygen species: An emerging approach for cancer therapy. Apoptosis 22: 1321-1335, 2017.

12. Bonekamp NA, Völkl A, Fahimi HD and Schrader M: Reactive oxygen species and peroxisomes: Struggling for balance. Biofactors 35: 346-355, 2009.

13. Fransen M, Nordgren M, Wang B and Apanasets O: Role of peroxisomes in ROS/RNS-metabolism: Implications for human disease. Biochimica et biophysica Biochim Biophys Acta 1822: 1363-1373, 2012

14. Kubota C, Torii S, Hou N, Saito N, Yoshimoto Y, Imai H and Takeuchi T: Constitutive reactive oxygen species generation from autophagosome/lysosome in neuronal oxidative toxicity. J Biol Chem 285: 667-674, 2010.

15. Santos CX, Tanaka LY, Wosniak J and Laurindo FR: Mechanisms and implications of reactive oxygen species generation during the unfolded protein response: Roles of endoplasmic reticulum oxidoreductases, mitochondrial electron transport, and NADPH oxidase. Antioxid Redox Signal 11: 2409-2427, 2009.

16. Angelova PR and Abramov AY: Role of mitochondrial ROS in the brain: From physiology to neurodegeneration. FEBS Lett 592: 692-702, 2018.

17. Oyewole AO and Birch-Machin MA: Mitochondria-targeted antioxidants. FASEB J 29: 4766-4771, 2015.

18. Halliwell B: Oxidative stress and neurodegeneration: Where are we now? J Neurochem 97: 1634-1658, 2006.

19. Ohsawa I, Ishikawa M, Takahashi K, Watanabe M, Nishimaki K, Yamagata K, Katsura K, Katayama Y, Asoh S and Ohta S: Hydrogen acts as a therapeutic antioxidant by selectively reducing cytotoxic oxygen radicals. Nat Med 13: 688-694, 2007.

20. Prabhakar NR: Sensory plasticity of the carotid body: Role of reactive oxygen species and physiological significance. Respir Physiol Neurobiol 178: 375-380, 2011.

21. Zhao RZ, Jiang S, Zhang L and Yu ZB: Mitochondrial electron transport chain, ROS generation and uncoupling (Review). Int J Mol Med 44: 3-15, 2019.

22. Khan SA, Nanduri J, Yuan G, Kinsman B, Kumar GK, Joseph J, Kalyanaraman B and Prabhakar R: NADPH oxidase 2 mediates intermittent hypoxia-induced mitochondrial complex I inhibition: Relevance to blood pressure changes in rats. Antioxid Redox Signal 14: 533-542, 2011.

23. Kang PT, Chen CL, Lin P, Zhang L, Zweier JL and Chen YR: Mitochondrial complex I in the post-ischemic heart: Reperfusion-mediated oxidative injury and protein cysteine sulfonation. J Mol Cell Cardiol 121: 190-204, 2018.

24. Yuan G, Adhikary G, McCormick AA, Holcroft JJ, Kumar GK and Prabhakar NR: Role of oxidative stress in intermittent hypoxia-induced immediate early gene activation in rat $\mathrm{PC} 12$ cells. J Physiol 557: 773-783, 2004

25. Higa A and Chevet E: Redox signaling loops in the unfolded protein response. Cell Signal 24: 1548-1555, 2012.

26. Görlach A, Bertram K, Hudecova S and Krizanova O: Calcium and ROS: A mutual interplay. Redox Biol 6: 260-271, 2015.

27. Bhandary B, Marahatta A, Kim HR and Chae HJ: An involvement of oxidative stress in endoplasmic reticulum stress and its associated diseases. Int J Mol Sci 14: 434-456, 2012.

28. Yong J, Bischof H, Burgstaller S, Siirin M, Murphy A, Malli R and Kaufman RJ: Mitochondria supply ATP to the ER through a mechanism antagonized by cytosolic $\mathrm{Ca}^{2}$. Elife 8: pii: e49682, 2019.

29. Malhotra JD and Kaufman RJ: Endoplasmic reticulum stress and oxidative stress: A vicious cycle or a double-edged sword? Antioxid Redox Signal 9: 2277-2293, 2007.

30. Mello T, Zanieri F, Ceni E and Galli A: Oxidative stress in the healthy and wounded hepatocyte: A cellular organelles perspective. Oxid Med Cell Longev 2016: 8327410, 2016.

31. Tu BP and Weissman JS: The FAD- and $\mathrm{O}(2)$-dependent reaction cycle of Erol-mediated oxidative protein folding in the endoplasmic reticulum. Mol Cell 10: 983-994, 2002.

32. Panday A, Sahoo MK, Osorio D and Batra S: NADPH oxidases: An overview from structure to innate immunity-associated pathologies. Cell Mol Immunol 12: 5-23, 2015.

33. Kaur G, Sharma A, Guruprasad K and Pati PK: Versatile roles of plant NADPH oxidases and emerging concepts. Biotechnol Adv 32: 551-563, 2014.

34. Konior A, Schramm A, Czesnikiewicz-Guzik M and Guzik TJ: NADPH oxidases in vascular pathology. Antioxid Redox Signal 20: 2794-2814, 2014 
35. Nisimoto $\mathrm{Y}$, Motalebi S, Han $\mathrm{CH}$ and Lambeth JD: The p67(phox) activation domain regulates electron flow from NADPH to flavin in flavocytochrome b(558). J Biol Chem 274: 22999-23005, 1999

36. Li L, Ren F, Qi C, Xu L, Fang Y, Liang M, Feng J, Chen B, Ning $\mathrm{W}$ and Cao J: Intermittent hypoxia promotes melanoma lung metastasis via oxidative stress and inflammation responses in a mouse model of obstructive sleep apnea. Respir Res 19: 28 , 2018.

37. Del Ben M, Fabiani M, Loffredo L, Polimeni L, Carnevale R, Baratta F, Brunori M, Albanese F, Augelletti T, Violi F and Angelico F: Oxidative stress mediated arterial dysfunction in patients with obstructive sleep apnoea and the effect of continuous positive airway pressure treatment. BMC Pulm Med 12: 36, 2012

38. Schulz R, Murzabekova G, Egemnazarov B, Kraut S, Eisele HJ, Dumitrascu R, Heitmann J, Seimetz M, Witzenrath M, Ghofrani HA, et al: Arterial hypertension in a murine model of sleep apnea: Role of NADPH oxidase 2. J Hypertens 32: 300-305, 2014.

39. Nair D, Dayyat EA, Zhang SX, Wang Y and Gozal D: Intermittent hypoxia-induced cognitive deficits are mediated by NADPH oxidase activity in a murine model of sleep apnea. PLoS One 6 : e19847, 2011

40. Nair D, Ramesh V and Gozal D: Adverse cognitive effects of high-fat diet in a murine model of sleep apnea are mediated by NADPH oxidase activity. Neuroscience 227: 361-369, 2012

41. Reddy JK and Mannaerts GP: Peroxisomal lipid metabolism. Annu Rev Nutr 14: 343-370, 1994.

42. Lodhi IJ and Semenkovich CF: Peroxisomes: A nexus for lipid metabolism and cellular signaling. Cell Metab 19: 380-392, 2014

43. Suzuki J: Short-duration intermittent hypoxia enhances endurance capacity by improving muscle fatty acid metabolism in mice. Physiol Rep 4: pii: e12744, 2016.

44. Cantu-Medellin N and Kelley EE: Xanthine oxidoreductase-catalyzed reactive species generation: A process in critical need of reevaluation. Redox Biol 1: 353-358, 2013.

45. Harris CM and Massey V: The reaction of reduced xanthine dehydrogenase with molecular oxygen. Reaction kinetics and measurement of superoxide radical. J Biol Chem 272: 8370-8379, 1997.

46. Harris CM and Massey V: The oxidative half-reaction of xanthine dehydrogenase with NAD; Reaction kinetics and steady-state mechanism. J Biol Chem 272: 28335-28341, 1997.

47. Wang S, Li Y, Song X, Wang X, Zhao C, Chen A and Yang P Febuxostat pretreatment attenuates myocardial ischemia/reperfusion injury via mitochondrial apoptosis. J Transl Med 13: 209, 2015.

48. Nanduri J, Vaddi DR, Khan SA, Wang N, Makerenko V and Prabhakar NR: Xanthine oxidase mediates hypoxia-inducible factor- $2 \alpha$ degradation by intermittent hypoxia. PLoS One 8 : e75838, 2013

49. Morgan BJ, Bates ML, Rio RD, Wang Z and Dopp JM: Oxidative stress augments chemoreflex sensitivity in rats exposed to chronic intermittent hypoxia. Respir Physiol Neurobiol 234: 47-59, 2016.

50. El Solh AA, Saliba R, Bosinski T, Grant BJ, Berbary E and Miller N: Allopurinol improves endothelial function in sleep apnoea: A randomised controlled study. Eur Respir J 27 997-1002, 2006.

51. Griscavage JM, Rogers NE, Sherman MP and Ignarro LJ: Inducible nitric oxide synthase from a rat alveolar macrophage cell line is inhibited by nitric oxide. J Immunol 151: 6329-6337, 1993.

52. Kumar A, Chen SH, Kadiiska MB, Hong JS, Zielonka J, Kalyanaraman B and Mason RP: Inducible nitric oxide synthase is key to peroxynitrite-mediated, LPS-induced protein radical formation in murine microglial BV2 cells. Free Radic Biol Med 73: 51-59, 2014

53. Contestabile A: Role of nitric oxide in cerebellar development and function: Focus on granule neurons. Cerebellum 11: 50-61, 2012.

54. Li S, Hafeez A, Noorulla F, Geng X, Shao G, Ren C, Lu G, Zhao H, Ding Y and Ji X: Preconditioning in neuroprotection: From hypoxia to ischemia. Prog Neurobiol 157: 79-91, 2017.

55. Yuan X, Guo X, Deng Y, Zhu D, Shang J and Liu H: Chronic intermittent hypoxia-induced neuronal apoptosis in the hippocampus is attenuated by telmisartan through suppression of iNOS/NO and inhibition of lipid peroxidation and inflammatory responses. Brain Res 1596: 48-57,2015.

56. Varadharaj S, Porter K, Pleister A, Wannemacher J, Sow A, Jarjoura D, Zweier JL and Khayat RN: Endothelial nitric oxide synthase uncoupling: A novel pathway in OSA induced vascular endothelial dysfunction. Respir Physiol Neurobiol 207: 40-47, 2015 .
57. Sun GY, Shelat PB, Jensen MB, He Y, Sun AY and Simonyi A Phospholipases A2 and inflammatory responses in the central nervous system. Neuromolecular Med 12: 133-148, 2010.

58. Akiba $\mathrm{S}$ and Sato T: Cellular function of calcium-independent phospholipase A2. Biol Pharm Bull 27: 1174-1178, 2004.

59. Radogna F, Sestili P, Martinelli C, Paolillo M, Paternoster L, Albertini MC, Accorsi A, Gualandi G and Ghibelli L: Lipoxygenase-mediated pro-radical effect of melatonin via stimulation of arachidonic acid metabolism. Toxicol Appl Pharmacol 238: 170-177, 2009.

60. Rordorf G, Uemura Y and Bonventre JV: Characterization of phospholipase A2 (PLA2) activity in gerbil brain: Enhanced activities of cytosolic, mitochondrial, and microsomal forms after ischemia and reperfusion. J Neurosci 11: 1829-1836, 1991.

61. Stephenson D, Rash K, Smalstig B, Roberts E, Johnstone E, Sharp J, Panetta J, Little S, Kramer R and Clemens J: Cytosolic phospholipase A2 is induced in reactive glia following different forms of neurodegeneration. Glia 27: 110-128, 1999.

62. Arai K, Ikegaya Y, Nakatani Y, Kudo I, Nishiyama N and Matsuki N: Phospholipase A2 mediates ischemic injury in the hippocampus: A regional difference of neuronal vulnerability. Eur J Neurosci 13: 2319-2323, 2001.

63. Lauritzen I, Heurteaux C and Lazdunski M: Expression of group II phospholipase A2 in rat brain after severe forebrain ischemia and in endotoxic shock. Brain Res 651: 353-356, 1994.

64. Elliot-Portal E,Laouafa S, Arias-Reyes C, Janes TA, Joseph V and Soliz J: Brain-derived erythropoietin protects from intermittent hypoxia-induced cardiorespiratory dysfunction and oxidative stress in mice. Sleep: 41, 2018 doi: 10.1093/sleep/zsy072.

65. Kim YS, Kwak JW, Lee KE, Cho HS, Lim SJ, Kim KS, Yang HS and Kim HJ: Can mitochondrial dysfunction be a predictive factor for oxidative stress in patients with obstructive sleep apnea? Antioxid Redox Signal 21: 1285-1288, 2014.

66. Slonkova J, Bar M, Nilius P, Berankova D, Salounova D and Sonka K: Spontaneous improvement in both obstructive sleep apnea and cognitive impairment after stroke. Sleep Med 32: 137-142, 2017.

67. Quan SF, Chan CS, Dement WC, Gevins A, Goodwin JL, Gottlieb DJ, Green S, Guilleminault C, Hirshkowitz M, Hyde PR, et al: The association between obstructive sleep apnea and neurocognitive performance-the Apnea Positive Pressure Long-term Efficacy Study (APPLES). Sleep 34: 303-314B, 2011.

68. Gagnon K, Baril AA, Gagnon JF, Fortin M, Décary A, Lafond C, Desautels A, Montplaisir J and Gosselin N: Cognitive impairment in obstructive sleep apnea. Pathol Biol (Paris) 62: 233-240, 2014

69. Xu LH, Xie H, Shi ZH, Du LD, Wing YK, Li AM, Ke Y and Yung WH: Critical role of endoplasmic reticulum stress in chronic intermittent Hypoxia-induced deficits in synaptic plasticity and Long-term memory. Antioxid Redox Signal 23: 695-710, 2015

70. Xie H, Leung KL, Chen L, Chan YS, Ng PC, Fok TF, Wing YK, $\mathrm{Ke}$ Y, Li AM and Yung WH: Brain-derived neurotrophic factor rescues and prevents chronic intermittent hypoxia-induced impairment of hippocampal long-term synaptic plasticity. Neurobiol Dis 40: 155-162, 2010.

71. Feng J, Wu Q, Zhang D and Chen BY: Hippocampal impairments are associated with intermittent hypoxia of obstructive sleep apnea. Chin Med J (Engl) 125: 696-701, 2012

72. Milton VJ and Sweeney ST: Oxidative stress in synapse development and function. Dev Neurobiol 72: 100-110, 2012.

73. Lin WC, Huang CC, Chen HL, Chou KH, Chen PC, Tsai NW, Chen $\mathrm{MH}$, Friedman M, Lin HC and Lu CH: Longitudinal brain structural alterations and systemic inflammation in obstructive sleep apnea before and after surgical treatment. J Transl Med 14: 139,2016

74. O'Donoghue FJ, Wellard RM, Rochford PD, Dawson A, Barnes M, Ruehland WR, Jackson ML, Howard ME, Pierce RJ and Jackson GD: Magnetic resonance spectroscopy and neurocognitive dysfunction in obstructive sleep apnea before and after CPAP treatment. Sleep 35: 41-48, 2012.

75. Canessa N, Castronovo V, Cappa SF, Aloia MS, Marelli S, Falini A, Alemanno F and Ferini-Strambi L: Obstructive sleep apnea: Brain structural changes and neurocognitive function before and after treatment. Am J Respir Crit Care Med 183: 1419-1426, 2011

76. Torelli F, Moscufo N, Garreffa G, Placidi F, Romigi A, Zannino S, Bozzali M, Fasano F, Giulietti G, Djonlagic I, et al: Cognitive profile and brain morphological changes in obstructive sleep apnea. NeuroImage 54: 787-793, 2011. 
77. Nizari S, Carare RO, Romero IA and Hawkes CA: 3D reconstruction of the neurovascular unit reveals differential loss of cholinergic innervation in the cortex and hippocampus of the adult mouse brain. Front Aging Neurosci 11: 172, 2019.

78. Han Q,Li G, Ip MS, Zhang Y, Zhen Z, Mak JC and Zhang N: Haemin attenuates intermittent hypoxia-induced cardiac injury via inhibiting mitochondrial fission. J Cell Mol Med 22: 2717-2726, 2018.

79. Hull TD, Boddu R, Guo L, Tisher CC, Traylor AM, Patel B, Joseph R, Prabhu SD, Suliman HB, Piantadosi CA, et al: Heme oxygenase-1 regulates mitochondrial quality control in the heart. JCI Insight 1: e85817, 2016.

80. Delbarba A, Abate G, Prandelli C, Marziano M, Buizza L, Arce Varas N, Novelli A, Cuetos F, Martinez C, Lanni C, et al: Mitochondrial alterations in peripheral mononuclear blood cells from Alzheimer's disease and mild cognitive impairment patients. Oxid Med Cell Longev 2016: 5923938, 2016.

81. Petersen MH, Budtz-Jørgensen E, Sørensen SA, Nielsen JE Hjermind LE, Vinther-Jensen T, Nielsen SM and Nørremølle A: Reduction in mitochondrial DNA copy number in peripheral leukocytes after onset of Huntington's disease. Mitochondrion 17: 14-21, 2014.

82. Leuner K, Pantel J, Frey C, Schindowski K, Schulz K, Wegat T, Maurer K, Eckert A and Müller WE: Enhanced apoptosis, oxidative stress and mitochondrial dysfunction in lymphocytes as potential biomarkers for Alzheimer's disease. J Neural Transm Suppl: 207-215, 2007.

83. Ciccone MM, Scicchitano P, Zito A, Cortese F, Boninfante B, Falcone VA, Quaranta VN, Ventura VA, Zucano A, Di Serio F, et al: Correlation between inflammatory markers of atherosclerosis and carotid intima-media thickness in Obstructive Sleep Apnea. Molecules 19: 1651-1662, 2014.

84. Yang Q, Wang Y, Feng J, Cao J and Chen B: Intermittent hypoxia from obstructive sleep apnea may cause neuronal impairment and dysfunction in central nervous system: The potential roles played by microglia. Neuropsychiatr Dis Treat 9: 1077-1086, 2013.

85. Lee EJ, Heo W, Kim JY, Kim H, Kang MJ, Kim BR, Kim JH, Park DY, Kim CH, Yoon JH and Cho HJ: Alteration of inflammatory mediators in the upper and lower airways under chronic intermittent hypoxia: Preliminary animal study. Mediators Inflamm 2017: 4327237, 2017.

86. Ryan S, Taylor CT and McNicholas WT: Selective activation of inflammatory pathways by intermittent hypoxia in obstructive sleep apnea syndrome. Circulation 112: 2660-2667, 2005.

87. Morgan MJ and Liu ZG: Crosstalk of reactive oxygen species and NF- $\kappa$ B signaling. Cell Res 21: 103-115, 2011.

88. Schoonbroodt S, Ferreira V, Best-Belpomme M, Boelaert JR, Legrand-Poels S, Korner M and Piette J: Crucial role of the amino-terminal tyrosine residue 42 and the carboxyl-terminal PEST domain of I kappa B alpha in NF-kappa B activation by an oxidative stress. J Immunol 164: 4292-4300, 2000

89. Hayden MS and Ghosh S: Shared principles in NF-kappaB signaling. Cell 132: 344-362, 2008.

90. Fang L, Choudhary S, Zhao Y, Edeh CB, Yang C, Boldogh I and Brasier AR: ATM regulates NF- $\kappa$ B-dependent immediate-early genes via RelA Ser 276 phosphorylation coupled to CDK9 promoter recruitment. Nucleic Acids Res 42: 8416-8432, 2014.

91. Williams A and Scharf SM: Obstructive sleep apnea, cardiovascular disease, and inflammation-is NF-kappaB the key? Sleep Breath 11: 69-76, 2007.

92. Anrather J, Racchumi G and Iadecola C: NF-kappaB regulates phagocytic NADPH oxidase by inducing the expression of gp91phox. J Biol Chem 281: 5657-5667, 2006.

93. Vasconcelos AR, Yshii LM, Viel TA, Buck HS, Mattson MP, Scavone $\mathrm{C}$ and Kawamoto EM: Intermittent fasting attenuates lipopolysaccharide-induced neuroinflammation and memory impairment. J Neuroinflammation 11: 85, 2014.

94. Do K, Laing BT, Landry T, Bunner W, Mersaud N, Matsubara T, Li P, Yuan Y, Lu Q and Huang H: The effects of exercise on hypothalamic neurodegeneration of Alzheimer's disease mouse model. PLoS One 13: e0190205, 2018

95. Wang J, Ming H, Chen R, Ju JM, Peng WD, Zhang GX and Liu CF: CIH-induced neurocognitive impairments are associated with hippocampal $\mathrm{Ca}(2+)$ overload, apoptosis, and dephosphorylation of ERK1/2 and CREB that are mediated by overactivation of NMDARs. Brain Res 1625: 64-72, 2015

96. QiG,Mi Y,Wang Y,LiR,Huang S,Li X and Liu X: Neuroprotective action of tea polyphenols on oxidative stress-induced apoptosis through the activation of the TrkB/CREB/BDNF pathway and Keap1/Nrf2 signaling pathway in SH-SY5Y cells and mice brain. Food Funct 8: 4421-4432, 2017.
97. Yin X,Zhang X,Lv C,LiC,Yu Y,Wang X andHan F: Protocatechuic acid ameliorates neurocognitive functions impairment induced by chronic intermittent hypoxia. Sci Rep 5: 14507, 2015.

98. Cervellati F, Cervellati C, Romani A, Cremonini E, Sticozzi C, Belmonte G, Pessina F and Valacchi G: Hypoxia induces cell damage via oxidative stress in retinal epithelial cells. Free Radic Res 48: 303-312, 2014.

99. Semenza GL and Prabhakar NR: HIF-1-dependent respiratory, cardiovascular, and redox responses to chronic intermittent hypoxia. Antioxid Redox Signal 9: 1391-1396, 2007.

100. Jung SN, Yang WK, Kim J, Kim HS, Kim EJ, Yun H, Park H, Kim SS, Choe W, Kang I and Ha J: Reactive oxygen species stabilize hypoxia-inducible factor-1 alpha protein and stimulate transcriptional activity via AMP-activated protein kinase in DU145 human prostate cancer cells. Carcinogenesis 29: 713-721, 2008.

101. Rabinovitch RC, Samborska B, Faubert B, Ma EH, Gravel SP, Andrzejewski S, Raissi TC, Pause A, St-Pierre J and Jones RG: AMPK maintains cellular metabolic homeostasis through regulation of mitochondrial reactive oxygen species. Cell Rep 21: $1-9,2017$

102. Guo H, Cao J, Li J, Yang X, Jiang J, Feng J, Li S, Zhang J and Chen B: Lymphocytes from intermittent hypoxia-exposed rats increase the apoptotic signals in endothelial cells via oxidative and inflammatory injury in vitro. Sleep Breath 19: 969-976, 2015.

103. Bianchi G, Di Giulio C, Rapino C, Rapino M, Antonucci A and Cataldi A: p53 and p66 proteins compete for hypoxia-inducible factor 1 alpha stabilization in young and old rat hearts exposed to intermittent hypoxia. Gerontology 52: 17-23, 2006.

104. da Rosa DP, Forgiarini LF, e Silva MB, Fiori CZ, Andrade CF, Martinez D and Marroni NP: Antioxidants inhibit the inflammatory and apoptotic processes in an intermittent hypoxia model of sleep apnea. Inflamm Res 64: 21-29, 2015.

105. Pan W and Kastin AJ: Can sleep apnea cause Alzheimer's disease? Neurosci Biobehav Rev 47: 656-669, 2014.

106. Andrade AG, Bubu OM, Varga AW and Osorio RS: The relationship between obstructive sleep apnea and Alzheimer's disease. J Alzheimers Dis 64 (Suppl 1): S255-S270, 2018.

107. Casagrande R, Stern P, Diehn M, Shamu C, Osario M,Zúñiga M, Brown PO and Ploegh H: Degradation of proteins from the ER of S. cerevisiae requires an intact unfolded protein response pathway. Mol cell 5: 729-735, 2000.

108. Walter P and Ron D: The unfolded protein response: From stress pathway to homeostatic regulation. Science 334: 1081-1086, 2011.

109. Szegezdi E, Logue SE, Gorman AM and Samali A: Mediators of endoplasmic reticulum stress-induced apoptosis. EMBO Rep 7: 880-885, 2006.

110. Chou YT, Zhan G, Zhu Y, Fenik P, Panossian L, Li Y, Zhang J and Veasey S: C/EBP homologous binding protein (CHOP) underlies neural injury in sleep apnea model. Sleep 36: 481-492, 2013.

111. Yao ZH, Kang X, Yang L, Niu Y, Lu Y, Gong CX, Tian Q and Wang JZ: Phenylbutyric acid protects against spatial memory deficits in a model of repeated electroconvulsive therapy. Curr Neurovasc Res 11: 156-167, 2014.

112. Nosyreva E and Kavalali ET: Activity-dependent augmentation of spontaneous neurotransmission during endoplasmic reticulum stress. J Neurosci 30: 7358-7368, 2010.

113. Archbold KH, Borghesani PR, Mahurin RK, Kapur VK and Landis CA: Neural activation patterns during working memory tasks and OSA disease severity: Preliminary findings. J Clin Sleep Med 5: 21-27, 2009.

114. Miller JF, Neufang M, Solway A, Brandt A, Trippel M, Mader I, Hefft S, Merkow M, Polyn SM, Jacobs J, et al: Neural activity in human hippocampal formation reveals the spatial context of retrieved memories. Science 342: 1111-1114, 2013.

115. Pena F and Ramirez JM: Hypoxia-induced changes in neuronal network properties. Mol Neurobiol 32: 251-283, 2005.

116. Clark RS, Kochanek PM, Dixon CE, Chen M, Marion DW, Heineman S, DeKosky ST and Graham SH: Early neuropathologic effects of mild or moderate hypoxemia after controlled cortical impact injury in rats. J Neurotrauma 14: 179-189, 1997.

117. Fung SJ, Xi MC, Zhang JH, Sampogna S, Yamuy J, Morales FR and Chase MH: Apnea promotes glutamate-induced excitotoxicity in hippocampal neurons. Brain Res 1179: 42-50, 2007.

118. Socodato R, Portugal CC, Rodrigues A, Henriques J, Rodrigues C, Figueira C and Relvas JB: Redox tuning of $\mathrm{Ca}^{2+}$ signaling in microglia drives glutamate release during hypoxia. Free Radic Biol Med 118: 137-149, 2018 
119. Macey PM, Sarma MK, Nagarajan R, Aysola R, Siegel JM, Harper RM and Thomas MA: Obstructive sleep apnea is associated with low GABA and high glutamate in the insular cortex. J Sleep Res 25: 390-394, 2016.

120. Opie GM, Catcheside PG, Usmani ZA, Ridding MC and Semmler JG: Motor cortex plasticity induced by theta burst stimulation is impaired in patients with obstructive sleep apnoea. Eur J Neurosci 37: 1844-1852, 2013

121. Gozal D, Nair D and Goldbart AD: Physical activity attenuates intermittent hypoxia-induced spatial learning deficits and oxidative stress. Am J Respir Crit Care Med 182: 104-112, 2010.

122. Toraldo DM, Di Michele L, Ralli M, Arigliani M, Passali GC, De Benedetto $\mathbf{M}$ and Passali D: Obstructive sleep apnea syndrome in the pediatric age: The role of the pneumologist. Eur Rev Med Pharmacol Sci 23 (1 Suppl): S15-S18, 2019.

123.Li Y, Ye J, Han D, Zhao D, Cao X, Orr J, Jen R, Deacon-Diaz N, Sands SA, Owens R and Malhotra A: The effect of upper airway surgery on loop gain in obstructive sleep apnea. J Clin Sleep Med 15: 907-913, 2019.
124. Epstein LJ, Kristo D, Strollo PJ Jr, Friedman N, Malhotra A Patil SP, Ramar K, Rogers R, Schwab RJ, Weaver EM, et al: Clinical guideline for the evaluation, management and long-term care of obstructive sleep apnea in adults. J Clin Sleep Med 5: 263-276, 2009.

125.Carlucci A, Ceriana P, Mancini M, Cirio S, Pierucci P, D'Artavilla Lupo N, Gadaleta F, Morrone E and Fanfulla F: Efficacy of Bilevel-auto treatment in patients with obstructive sleep apnea not responsive to or intolerant of continuous positive airway pressure ventilation. J Clin Sleep Med 11: 981-985, 2015.

(i) $(-)$ This work is licensed under a Creative Commons Attribution-NonCommercial-NoDerivatives 4.0 International (CC BY-NC-ND 4.0) License. 